Instituto Internacional de Investigación y Desarrollo Tecnológico Educativo INDTEC, C.A.

DOI: https://doi.org/10.29394/Scientific.issn.2542-2987.2020.5.18.9.173-192

OAI-PMH: http://www.indteca.com/ojs/index.php/Revista Scientific/oai

Artículo Original / Original Article

\title{
Inteligencia Social en la Comunicación: Una Aproximación Teórica para la Gerencia Universitaria
}

Autora: María Auxiliadora Campos Medina Universidad Nacional Abierta, UNA mariauxicamposmedina@hotmail.com Barquisimeto, Venezuela https://orcid.org/0000-0002-1370-4491

\section{Resumen}

Las universidades, como entes propulsores de desarrollo social y conocimiento requieren de una gerencia que en la praxis propicien una comunicación más efectiva a través del manejo de la inteligencia social. La presente investigación persigue la construcción teórica respecto al manejo de la inteligencia social como medio en la comunicación de la gerencia en la Universidad Centroccidental Lisandro Alvarado (UCLA) y la Universidad Nacional Abierta (UNA) dentro de un contexto ontológico complejo. La perspectiva metodológica usada fue el interpretativismo. Se direccionó por el método fenomenológico-hermenéutico. Se utilizó la técnica de entrevista a profundidad a los rectores de estas dos universidades venezolanas, como a grupos focales conformados por seis decanos. El tratamiento de la información fue sometido a un proceso de análisis para el establecimiento de las categorías, sus dimensiones y la triangulación. Esto permitió comprender la gestión de la inteligencia social en la comunicación en las universidades. Se concluye que estos gerentes han ejecutado efectivamente su rol desde el punto de vista tradicional, sin embargo, mostraron apertura a un nuevo proceso comunicacional. Deben afinar las destrezas de la inteligencia emocional. Este estudio condujo a la construcción del referente teórico sobre inteligencia social en la comunicación para la gerencia universitaria, el cual llamaremos INAPAP.

Palabras clave: gerencia; inteligencia social; comunicación.

Cómo citar este artículo:

Campos, M. (2020). Inteligencia Social en la Comunicación: Una Aproximación Teórica para la Gerencia Universitaria. Revista Scientific, 5(18), 173-192, e-ISSN: 2542-2987. Recuperado de: https://doi.org/10.29394/Scientific.issn.2542-2987.2020.5.18.9.173-192

Fecha de Recepción: 25-05-2020
Fecha de Aceptación: 13-08-2020
Fecha de Publicación: 05-11-2020 
OAI-PMH: http://www.indteca.com/ojs/index.php/Revista_Scientific/oai

Artículo Original / Original Article

\title{
Social Intelligence in Communication: A Theoretical Approach for University Management
}

\begin{abstract}
Universities, as propellants of social development and knowledge, require a management that, in practice, encourages more effective communication through the management of social intelligence. The present investigation pursues the theoretical construction regarding the management of social intelligence as a means in the communication of the management at the Universidad Centroccidental Lisandro Alvarado (UCLA) and the Universidad Nacional Abierta (UNA) within a complex ontological context. The methodological perspective used was interpretivism. It was directed by the phenomenological-hermeneutical method. The in-depth interview technique was used with the rectors of these two Venezuelan universities, as well as with focus groups made up of six deans. The information treatment was subjected to an analysis process for the establishment of categories, their dimensions and triangulation. This allowed us to understand the management of social intelligence in communication in universities. It is concluded that these managers have effectively executed their role from the traditional point of view, however, they showed openness to a new communicational process. They must hone the skills of emotional intelligence. This study led to the construction of the theoretical reference on social intelligence in communication for university management, which we will call INAPAP.
\end{abstract}

Keywords: management; social intelligence; communication.

How to cite this article:
Campos, M. (2020). Social Intelligence in Communication: A Theoretical Approach for University
Management. Revista Scientific, 5(18), 173-192, e-ISSN: 2542-2987. Recovered from:
https://doi.org/10.29394/Scientific.issn.2542-2987.2020.5.18.9.173-192

Date Received:

Date Acceptance:

25-05-2020

13-08-2020

Date Publication:

05-11-2020 


\section{Introducción}

Entre los avances obtenidos por la gerencia universitaria se encuentra la humanización de sus relaciones, dejando atrás los viejos esquemas gerenciales, en donde el gerente, revestido de poder, mantenía distancias infranqueables con sus seguidores y sus necesidades, las mismas que atañen a la organización. En la actualidad el enfoque de la gestión gerencial se perfila hacia el apropio de estrategias para transformarlas en algo más humano, donde el talento es considerado fuente inagotable de conocimiento.

Las universidades como entes organizacionales proveedoras de progreso generan expectativas en cuanto a su capacidad de adaptación a los vertiginosos cambios que provienen de una sociedad ciberglobalizada. Por lo tanto, se requiere una gerencia universitaria que redimensione la forma cómo se ejecutan las interrelaciones sociales en la organización, con la inclusión de la perfecta conexión entre la razón y la emoción.

Desde el punto de vista praxeológico y axiológico, la incorporación de la inteligencia social en los procesos comunicativos de las universidades, basada en el correcto manejo de la empatía, del autocontrol y el manejo de las emociones ajenas, la comunicación clara, enmarcados en valores como el respeto y la cooperación, hará de la gerencia un ente apto en el uso de la autoridad, con influencia para orquestar soluciones en pro del bienestar de los miembros de la comunidad universitaria y una mejor proyección de la misma hacia el entorno.

En base a lo planteado, surgió la inquietud de reflexionar acerca de la imperiosa necesidad que tiene la gerencia de la Universidad Centroccidental Lisandro Alvarado (UCLA) y la Universidad Nacional Abierta (UNA), en ejecutar cambios a nivel individual, para que los mismos se proyecten en el seno de la institución. En virtud de lo enunciado, se infiere en la presente investigación, que existe un vacío en la regulación de la empatía, componente esencial de la inteligencia social y de la comunicación, porque es el puente de 
conexión que tienen los gerentes con sus agremiados, de ella surgen la alineación y cooperación para los proyectos corporativos.

Al respecto, Chiavenato (2009): aporta que el gerente inteligente socialmente debe poseer la capacidad de adaptación a la crisis manteniéndose en equilibrio con sus emociones. Esto depende de las herramientas usadas y de qué modo se aplican. Más allá de cualquier modo de gestión es conveniente recordar que el talento humano es lo que otorga valor agregado a las organizaciones.

En tal sentido, el gerente que sea capaz de empatizar, ejecutar transformaciones pertinentes y profundas, de comunicarse de manera clara podrá afrontar con éxito la más fuerte crisis, porque todas estas aptitudes atraerán seguidores con deseos de formar equipos proactivos, responsables, con sentido de pertenencia a la organización, que perseveran hasta lograr los objetivos propuestos en beneficio propio y de la institución.

Bajo estas premisas, tomando en cuenta lo actual y novedoso que resulta el tema de la inteligencia social en la comunicación de la gerencia universitaria, fiel al cumplimiento de las intencionalidades de estudio se justifica esta aproximación teórica, que representa un gran aporte a las universidades venezolanas.

Como antecedente a este interesante estudio tenemos la investigación de corte teórico-documental, con diseño bibliográfico, técnica documental, realizada por Loaiza y Pirela (2015): cuyo objetivo fue analizar el liderazgo en organizaciones venezolanas, evidenció la necesidad de un liderazgo integrador con visión de transformación que propicie la armonía organizacional con base en la comunicación, que infunda confianza y la motivación a la búsqueda del conocimiento.

Para los filósofos, la inteligencia, proviene del latín intelligentia, que a su vez procede de inteligere, era la propia acción del intelecto, el proceso de 
conocimiento e interiorización de la realidad. Ya en el siglo XIX, emergen nuevos avances con respecto a esta facultad humana, entre ellos la del afamado psicólogo estadounidense Thorndike (1920): quien enfocó su teoría en la existencia de tres tipos de inteligencia: abstracta, mecánica y social.

En otro orden de ideas, es oportuno mencionar que al igual que la inteligencia, las emociones han ocupado un lugar privilegiado en los estudios científicos, de los cuales han surgido diversas corrientes, sobre todo en relación al éxito y la regulación de las mismas, de este modo gestionar las relaciones sociales.

En tal sentido, las emociones son de gran funcionalidad social, por ser una fuente de motivación, a su vez sirven de canalizadores de los juicios u opiniones de los demás. Son concebidas por Orón (2019): como una plataforma otorgada por la naturaleza con el fin obtener crecimiento personal, que a su vez son controladas por la cognición

Por otra parte, las definiciones tradicionales de la inteligencia hacen referencia a los aspectos cognitivos, como memoria, capacidad de resolver problemas. Sin embargo, los estudios más recientes apuntan a dar importancia a las emociones. En igual dirección, Goleman (2000a): realizó valiosos aportes científicos que dieron apertura a estudios sobre la dicotomía inteligenciaemoción.

Por otra parte, Albrecht (2007): quien es el autor del libro Inteligencia Social, concibe este tipo de inteligencia como una fusión de habilidades como comprensión básica de la gente, conciencia social estratégica y la aptitud para lograr éxito en la interacción social. Es la capacidad para llevarse bien con la gente y lograr que cooperen en el intento de conocer su comportamiento social.

La inteligencia social hace de una organización, una corporación inteligente, a través de un gerente que pueda resolver sus problemas de gestión y la prosperidad. Se requiere un gerente con habilidades para motivar 
a sus empleados al logro de metas reales, con capacidad de comunicación: la utiliza para el logro de aprendizajes sociales significativos y, cuando estos aprendizajes son compartidos entre varios sujetos, se está en presencia de la inteligencia social, que equivale al desarrollo colectivo en armonía con su entorno (ecología dialéctica).

Según Goleman (2000b): las actitudes que debe poseer un gerente con inteligencia social son: empatía que significa comprender a los demás, orientación al servicio, ayudar a los demás a desarrollarse. Aunado a esto, tiene destrezas para una comunicación eficaz, que contiene elementos como: asertividad, exteriorización de sus emociones, escucha empática a sus empleados, comparte críticas constructivas, habilidad para resolver conflictos de grupos.

Uno de los modelos de inteligencia social más divulgados es el de Goleman (2000c): este destacado investigador manifiesta que la inteligencia social es la relación recíproca entre emociones del gerente y sus empleados reflejada en la armonía social y organizacional. Su modelo consta de varios componentes, que se traslucen en el éxito gerencial. Estos son: empatía, aprovechamiento de la diversidad, influencia, manejo de conflictos y ser catalizadores de cambio.

En el transcurso de la historia, surgieron determinantes estudios acerca de la empatía. En este sentido, Goleman (2000d): la concibe como radar social, con ella comprendemos al otro, logramos entender sus sentimientos. Es una brújula que orienta al conocimiento de la postura del otro.

En otro sentido, Bateman y Snell (2005): sostienen que un gerente debe seguir cuatro etapas, como estrategia de resolución de conflictos en la organización. En primer lugar, indagar sobre los motivos de la controversia con entrevista a los involucrados, en segundo lugar, buscar soluciones creativas con la participación del personal. El tercer paso es ejecutar acciones con el fin de evitar posibles repeticiones del conflicto. Hacer revisiones periódicas de la 
situación problemática, para supervisar los resultados y realizar correctivos.

Con respecto al siguiente componente del modelo de inteligencia social concebido como aprovechar la diversidad, Goleman (2000e): infiere que una amenaza contra el éxito organizacional son los estereotipos grupales porque pueden destruir la cohesión y el logro de metas organizacionales por la difusión de patrones culturales y estigmas negativos. Estos, que a su vez perjudican sobremanera los grupos minoritarios de una organización, pueden repercutir de manera negativa por poseer un poder emocional muy fuerte

En cuanto a la influencia como componente de la inteligencia social, conforme a Goleman (2000f): es uno de los teóricos que más ha aportado al avance de las organizaciones con su teoría de las inteligencias emocional y social, sostiene que un líder con esta capacidad se caracteriza por saber despertar emociones específicas en el otro, respeto por el cargo que ejercen, pasión por un proyecto, competencia para superar retos y producciones de otras organizaciones o dominar las emociones de un trabajador enojado por alguna injusticia.

Es necesario, por demás que el gerente, como catalizador de cambios, modele conductas que sean congruentes con sus pensamientos. En el caso de los gerentes de Universidades, deben estar abiertos al cambio puesto que deben ser entidades puentes de conocimientos que van a la par del avance de la ciencia y la tecnología. Este cambio debe ser planificado según las necesidades y prioridades. La transformación será más real y permanente cuando los colaboradores sientan que su potencial humano es tomado en cuenta, y sienta que los beneficios organizacionales obtenidos de estos cambios también repercutan en sus mejoras personales y laborales.

Por todo lo anteriormente argumentado, dada esta etapa de cambios dinámicos y vertiginosos, se hace pertinente una gerencia con un perfil transformacional. En este aspecto, Pérez-Ortega, Jiménez-Valdés y RomoMorales (2017): dicen que es la más idónea para afrontar los retos actuales 
que deben asumir las organizaciones universitarias en cuanto a sustentabilidad, auge vertiginoso de la tecnología, la escasez de recursos, de acuerdo a sus funciones misionales, a través de la fusión de la dicotomía teoría-ciencia, luego adoptar conductas de cambio, manifiestas en óptimas relaciones interpersonales, basadas en el respeto, la escucha empática y ser ejemplo a seguir.

Otra variable emergida en este estudio es la comunicación en la gerencia, la cual es concebida como un proceso dinámico, primordial de toda relación social, puesto que el mecanismo que regula y hace posible interacción entre las personas.

El término comunicación proviene del latín "comunicare" que significa poner en común, compartir un mensaje o información. Un gerente con inteligencia social debe manejar y conocer estrategias de comunicación organizacional que estén acordes con lo exigido por una sociedad del conocimiento cada vez más veloz y dinámica. De no adaptarse a estos nuevos procesos comunicacionales, actualmente más tecnológicos, pero más humanos a la vez, la información se verá más desvirtuada, con tendencia al rumor, a los fracasos en la búsqueda del objetivo en común y hacia conflictos organizativos

En este mismo orden de ideas, la comunicación abordada desde el punto de vista organizacional. De acuerdo al aporte de Quiñones, Hernández y Cordón (2020): la comunicación es un acto reflexivo, dialógico que conlleva a la transformación, dada por espacios de participación. En este sentido, la gerencia debe asumir los nuevos retos de acuerdo a los entornos socioeconómicos del mundo, con el propósito de incrementar la productividad y la calidad del conocimiento.

El objetivo principal de esta investigación es develar las habilidades de la inteligencia social en la comunicación de la gerencia a través de las voces de los actores sociales en las Universidades públicas del estado Lara, 
Venezuela, tales como la Universidad Centroccidental Lisandro Alvarado y la Universidad Nacional Abierta.

\section{Metodología (Materiales y métodos)}

La presente investigación parte del interpretativismo, emerge como reacción al intento de desarrollar una ciencia natural, basada en los fenómenos sociales. Se fundamenta en la extrapolación de la propia psique hacia la del ser al cual se trata de comprender. En este caso se permitió la aproximación de manera teórica hacia la realidad a través de la cosmovisión de los actores sociales en la gerencia de la Universidad Centroccidental Lisandro Alvarado (UCLA) y la Universidad Nacional Abierta (UNA), para analizar cómo se emplea la inteligencia social en la comunicación con sus empleados.

Esta investigación fue abordada desde la perspectiva del método Fenomenológico-Hermenéutico. Al respecto, Fuister (2019a): arguye que está dirigida hacia el proceso descriptivo e interpretativo de un fenómeno desde lo profundo de la experiencia humana, apela a la aproximación del sujeto para dar significado a su entorno y, a la fenomenología como un modo de sacar a la luz y hacer visible algo en sí mismo.

En el presente estudio se utilizó la entrevista a profundidad, como técnica de recolección de datos, por su idoneidad en la comprensión de las perspectivas que tienen las personas implicadas en la investigación acerca del fenómeno a estudiar. Esta fusiona tres aspectos: 1. Interpretación; 2. Compresión; y 3. entendimiento, lo cual le da un sentido fenomenológico, sustentado por la hermenéutica, porque tiene como base el comportamiento humano.

Por otra parte, la corriente que propició el posicionamiento del fenómeno investigado es la cualitativa, percibida por Sánchez (2019a): como aquella que permite el debate entre los hechos y la interpretación de los mismos, con apoyo en la búsqueda de información bibliográfica y fundamentos 
epistémicos fenomenológicos. Requiere que el sujeto en su rol de investigador se sumerja en el entorno social del fenómeno a estudiar para lograr sensibilizarse. Así pues, se constituye en una unidad de análisis, con la cual se investiga una cualidad específica, sus nexos y relaciones con el todo.

De acuerdo a los mencionados Fuister (2019b); y Sánchez (2019b): esta corriente se desarrolla en cuatro fases: la primera, llamada reflexiva, permite desarrollar el marco teórico y planificar las fases posteriores, Así como la identificación de los propósitos del estudio. La segunda es la etapa de campo. Esta permite al investigador el acceso progresivo a la información que requiere para su estudio De este modo, fueron seleccionados actores sociales, quienes con sus vivencias y experiencias facilitaron la aproximación teórica en el uso de la inteligencia social en la comunicación por parte de la gerencia de la Universidad Centroccidental Lisandro Alvarado y la Universidad Nacional Abierta.

La última etapa está representada por la elaboración, presentación y difusión del informe de la investigación. El diseño elegido para la presente investigación es el de estudio de casos, que en opinión de Yin (2013): es una descripción y análisis detallados de unidades sociales o entidades educativas únicas. Esta parte de la particularidad para abordar a la complejidad, en un caso particular, en circunstancias concretas.

Luego se procedió al proceso de categorización que en opinión de Cisterna (2005): es aquel en el cual surgen tópicos en sí mismos, de allí emergieron las subcategorías, que detallan dichos tópicos en micro aspectos, todo bajo la responsabilidad de la investigadora, después del proceso de construcción de las categorías, son organizadas y codificadas.

A continuación, se muestra una matriz con algunas muestras de las entrevistas realizadas a los actores sociales que contribuyeron con sus voces a la elaboración de las categorías orientadoras y las subcategorías con sus codificaciones pertinentes, que surgen de las iniciales de cada categoría 
orientadora. En el cuadro 1, se aprecia la matriz donde se sistematiza lo expresado por los actores sociales y las categorías concernientes.

Cuadro 1. Matriz para el proceso de categorización.

\begin{tabular}{|l|c|c|}
\hline Transcripción de la Entrevista & Categoría Orientadora & Codificación \\
\hline $\begin{array}{l}\text {...si yo digo que estoy de acuerdo con esto, él lo que } \\
\text { está buscando es la empatía, ósea la simpatía, la } \\
\text { identificación y no la objetividad entonces en ese } \\
\text { momento debo permanecer y permanezco inmutable } \\
\text { lo digo de frente y de frente es no o de frente es si y } \\
\text { no le doy ningún otro detalle porque entonces viene } \\
\text { un dialogo. }\end{array}$ & Inteligencia Social & $\begin{array}{c}\text { Inteligencia } \\
\text { Social } \\
\text { (I.S.) }\end{array}$ \\
$\begin{array}{l}\text { pes que simplemente hay un elemento de la } \\
\text { me preguntan porque no y tengo la oportunidad de } \\
\text { decirle que siento algunos elementos, la persona } \\
\text { comprende, que esos elementos fueron mal } \\
\text { expuestos o están errados y allí se va al dialogo } \\
\text { entonces una conversación por supuesto a recuerda } \\
\text { que a nivel gerencial. }\end{array}$ & Comunicación & Comunicación \\
\hline
\end{tabular}

Fuente: La Autora (2020).

Producto de lo sugerido por los actores sociales y del análisis de las categorías emergieron las correspondientes subcategorías, las cuales generaron la aproximación teórica planteada en esta investigación. Por lo consiguiente, de la categoría Inteligencia social, emergieron las siguientes subcategorías: empatía, catalizador de cambio, aprovechar la diversidad, autenticidad, generar oportunidades y manejo de emociones. Así mismo, luego del análisis de la categoría comunicación se determinó las siguientes subcategorías: comunicación abierta, claridad, escucha empática, uso de redes sociales y rapport. Para su organización se utilizó la técnica de la cromatización.

Para la recolección y análisis de datos se utilizó la técnica de triangulación, una herramienta para optimizar la validez de los resultados, se depura los vacíos del proceso investigativo que vienen de manera intrínseca cuando se utiliza un solo método de recogida de datos y la subjetividad del investigador. Se puede afirmar que, a mayor grado de triangulación, mayor es el grado de fiabilidad. Así se efectúa un proceso de comparación entre los 
datos aportados por los actores sociales, en este caso los informantes clave y el grupo focal.

En la presente investigación se realizó la triangulación con los datos obtenidos a través de los Actores sociales (rectores de la Universidad Centroccidental Lisandro Alvarado y de la Universidad Nacional Abierta) y los sujetos partícipes en los grupos focales ( 3 decanos y 3 Coordinadores de las mencionadas universidades respectivamente).

\section{Resultados (análisis e interpretación de los resultados)}

Como resultado del pensamiento analítico y su conexión con el estado cognoscente, afloró la construcción de una aproximación teórica, luego de un encuentro intersubjetivo con los actores sociales, que conlleva a emitir unas concepciones acerca de la inteligencia social en la comunicación en la gerencia. Bajo estas consideraciones, se hace menester que la gerencia universitaria se apropie y desarrolle ciertas sociales, evidenciado en los testimonios de los actores sociales y las teorías de diversos autores.

Esta inquietud fue parte de la motivación que dio origen a este constructo teórico, el emergió en base a dos componentes, que son palabras comunes a las categorías de la presente investigación: inteligencia social, comunicación. Emerge, a si también tres componentes que interrelacionan como un trípode para el éxito de la praxis gerencial universitaria, representados por la Interacción, la Aptitud y la Apertura, la cual toma como nombre "INAPAP".

La interacción social hace del gerente una persona capacitada para manejar sus emociones de manera correcta ante situaciones difíciles, para el debido discernimiento al momento de optar por la mejor solución, a través del reconocimiento de las necesidades básicas, incluidas las emocionales, base fundamental para la común-unidad de las partes. La gerencia universitaria desarrollaría interacciones sociales inteligentes si hiciera uso de la dicotomía 
razón-emoción combinada con el uso del poder.

En cuanto al componente aptitud, es un aspecto que enlaza a la inteligencia y la comunicación, puesto que el ser humano, a través del lenguaje transmite aspectos recónditos entre sus emociones y el pensamiento. En este sentido la comunicación gerencial, se discurre a través de dos canales: el superior, que transmite la racionalidad, las palabras y su sentido. El inferior enmarcado en el lenguaje no verbal, donde se refleja la sincronía del gerente con sus seguidores.

Es oportuno destacar que la destreza o aptitud de integrar la inteligencia y la comunicación funciona como calibrador emocional para el gerente ante las interacciones sociales en las organizaciones, para actuar con el justo tono emocional ante una confrontación, tomar las mejores decisiones, transmitir un mensaje claro para la comprensión y empatía entre gerente-trabajador.

En lo que respecta a la apertura, la inteligencia social es el conducto hacia el desarrollo de relaciones abiertas hacia el mundo del otro. A través de ésta y del ejercicio del poder y la influencia sobre los trabajadores, de un modo recíproco, la gerencia podrá sacar provecho de las oportunidades, propiciadas por la diversidad de personalidades y saberes, por la comprensión de las emociones que le contagian sus seguidores, visión estratégica y flexibilidad para hace suscitar soluciones creativas a través de la confianza que ésta ejerce sobre sus seguidores.

Se requiere de estas habilidades para lograr interacciones inteligentes por parte de la gerencia y dar apertura a nuevas estrategias de soluciones en la praxis gerencial, en la Universidad Centroccidental Lisandro Alvarado y la Universidad Nacional Abierta, como entes propulsores de conocimiento. Con la figura 1, se representa la conexión de las palabras emergidas en esta aproximación. 
Figura 1. Representación de la aproximación teórica: INAPAP.

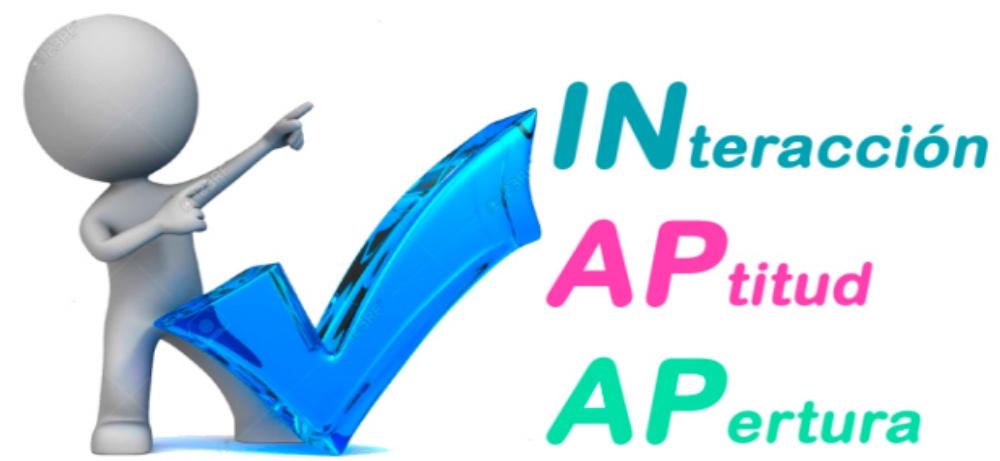

Fuente: Las Autora (2020).

\section{Conclusiones}

La investigación cualitativa, permitió, desde una perspectiva fenomenológica-hermenéutica, aflorar la creatividad reflexiva sobre los hallazgos referentes a la inteligencia social en la comunicación de la gerencia enfocada en la Universidad Centroccidental Lisandro Alvarado y LA Universidad Nacional Abierta. Este proceso dejó libertad al pensamiento deliberante, en el cual hubo confrontación entre lo develado por los actores sociales y la realidad existente.

Esta investigación conllevó a la interacción dialéctica del sujeto con el fenómeno de estudio con énfasis en el papel del pensamiento en la construcción del conocimiento y la comprensión de los fenómenos organizacionales.

El encuentro del investigador, en su rol de sujeto cognoscente y la realidad condujo a la reducción eidética del fenómeno estudiado, como principio cognitivo a través de las consideraciones que emergieron de la confrontación de tres aspectos: referentes gnoseológicos, realidad encontrada en la versión de los actores sociales y el análisis como producto reflexivo, dio como resultado la generación de una aproximación con connotación hacia la inteligencia social en la comunicación de la gerencia universitaria. 
Esta episteme hizo reflexionar acerca de la necesidad de una gestión gerencial más sinérgica hacia el desarrollo de relaciones interpersonales y laborales, con el balance de la dicotomía-razón-emoción, manifiesto en la comunicación, que por demás no es tarea fácil, aún más si no se cuenta con la ayuda de un equipo inteligente, con las mismas metas y con sentido de cooperación y pertenencia.

En este aspecto, este estudio concluye que la inteligencia social como una capacidad humana por la cual se establecen relaciones interpersonales más íntegras, con sensibilidad ante la realidad social y plena conciencia de las emociones propias y ajenas. Así mismo, esta habilidad, contribuye a una interrelación dialógica más clara y empática, con una postura, tono de voz y gestos adecuados, en el lugar y tiempo indicados.

Estas concepciones conllevan a considerar que el logro de interacciones inteligentes es requisito indispensable en la gerencia universitaria, como virtud humana, para transformar el entorno laboral en una plataforma hacia el buen entendimiento, la cooperación y la proactividad, a la hora de enfrentar los nuevos desafíos para las magnas casas de estudio, que se debaten entre una realidad nacional más agobiante y una sociedad globalizada cada más cambiante.

Por otra, es patente el hecho de que los actores sociales han ejecutado efectivamente su rol, desde un punto de vista tradicional, Muchas veces han tenido que utilizar un liderazgo autocrático, sin embargo, también han demostrado apertura a un proceso comunicacional donde se plasma la disposición de escuchar a los diferentes gremios que laboran en el recinto universitario. Se detectó las principales causas de los conflictos más frecuentes, como son: fallas en el cumplimiento de reglamentos y normas, déficit presupuestario, absentismo, entre otros. Si existe acercamiento entre las partes, habrá responsabilidad e identidad organizacional.

En otro aspecto, este estudio hace ostensible que existe una debilidad 
por parte de las autoridades universitarias y es la manera como conciben las relaciones empáticas, que por demás distan mucho de las relaciones ecpáticas, porque en las primeras el sujeto puede comprender y reaccionar proactivamente ante las preocupaciones de los demás que subyacen en las respuestas que esperan ante sus requerimientos, en este caso del gerente. La sintonía jefe-trabajador muchas veces es rechazada interiormente por parte de quien ejerce la autoridad para evitar involucrarse de manera emocional y así no mostrarse vulnerable.

Así mismo, lo expresado por los actores sociales, conlleva a reflexionar que la esencia de la empatía es la percepción de lo que otros sienten sin decirlo, cuestión contraria a la ecpatía que consiste en el rechazo de tal sensación. La capacidad de percibir comunicaciones sutiles, es requerida por los gerentes, de este modo perciben lo que realmente quieren decir los trabajadores, de esta forma evitarían manipulaciones, aspecto que produce frustración tanto por parte de las autoridades como de los empleados.

Desde otro punto de vista, es inequívoco la necesidad de afrontar, por parte de la gerencia universitaria, la brecha digital que existe en las universidades y la cibersociedad, de manera que las universidades puedan actualizar sus procesos comunicativos de manera interna y con su entorno, en el uso de las herramientas de la web 2.0, para el desarrollo profesional del cuerpo académico. Por todo lo manifestado anteriormente, es perentorio que las universidades proporcionen encuentros entre los docentes, las innovaciones tecnológicas y la adaptación a las necesidades de la sociedad actual.

En razón a lo expuesto, las universidades deben cruzar la frontera entre las organizaciones tradicionales, con autoridades que, aunque cumplen cabalmente su rol, se plasman distantes de las verdaderas necesidades y sentimientos, y aquellas que consideran las relaciones interpersonales inteligentes como un medio para ejercer un proceso comunicativo 
organizacional de manera clara y auténtica, con el calibre justo de las emociones propias y manejo de las relaciones ajenas, con lo cual se promueve la cultura de paz.

En torno a estos criterios, el pensamiento reflexivo permite comprender, las posturas de cada uno de los actores sociales, a fin de extraer sus significados emocionales a través de la óptica de sus vivencias. Subrayo que generar esta aproximación teórica, que por sus iniciales de Interacción, Aptitud y Apertura (INAPAP) es de un gran valor desde el punto de vista investigativo por ser un aporte para el manejo de la inteligencia social en la comunicación de la gerencia universitaria.

De esta manera, la aproximación teórica da respuesta a las interrogantes de inicio en este interesante estudio: ¿La inteligencia social repercute en la productividad de una organización?; ¿El gerente con inteligencia social realiza una comunicación más efectiva con sus seguidores?. Queda abierta como línea de investigación para futuras investigaciones.

\section{Referencias}

Albrecht, K. (2007). Inteligencia Social: La nueva ciencia del éxito. ISBN: 9788466629676. Barcelona, España: Ediciones B.

Bateman, T., \& Snell, S. (2005). Administración, liderazgo y colaboración en un mundo competitivo. ISBN: 978-970-10-7279-0. México: McGraw-Hill / Interamericana Editores, S.A.

Chiavenato, I. (2009). Gestión del talento humano. ISBN: 109701073401. México: McGraw-Hill.

Cisterna, F. (2005). Categorización y triangulación como procesos de validación del conocimiento en investigación cualitativa. Theoria, 14(1), 61-71, e-ISSN: 0717-196X. Recuperado de:

https://www.redalyc.org/articulo.oa?id=29900107

Fuister, D. (2019a,b). Investigación cualitativa: Método fenomenológico 
hermenéutico. Propósitos y Representaciones, 7(1), 201-229, e-ISSN: 2310-4635. Recuperado de:

http://dx.doi.org/10.20511/pyr2019.v7n1.267

Goleman, D. (2000a,b,c,d,e,f). La Inteligencia Emocional: Por qué es más importante que el cociente intelectual. ISBN: 9789684972056. Buenos Aires, Argentina: Javier Vergara Editor.

Loaiza, C., \& Pirela, L. (2015). Liderazgo en organizaciones venezolanas. Revista Venezolana de Gerencia, 20(69), 152-171, e-ISSN: 1315-9984. Recuperado de:

https://produccioncientificaluz.org/index.php/rvg/article/view/19707

Orón, J. (2019). Neuropsicología de las emociones. ISBN: 978-84-3684089-6. Madrid, España: Ediciones Pirámide.

Pérez-Ortega, G., Jiménez-Valdés, G., \& Romo-Morales, G. (2017). Caracterización del liderazgo transformacional de los directivos de instituciones de educación superior. Caso de estudio en una universidad del departamento de Antioquia (Colombia). Entramado, 13(1), 48-61, e-ISSN: 2539-0279. Recuperado de:

https://doi.org/10.18041/entramado.2017v13n1.25137

Quiñones, A., Hernández, I., \& Cordón F. (2020). La comunicación como elemento clave en la gestión de la informatización del proceso extensión universitaria. Revista de Desarrollo Sustentable, Negocios, Emprendimiento y Educación, 2(3), 1-11, e-ISSN: 2695-6098. Recuperado de:

https://www.eumed.net/rev/rilcoDS/03/informatizacion-extensionuniversitaria.html

Sánchez, F. (2019a,b). Fundamentos Epistémicos de la Investigación Cualitativa y Cuantitativa: Consensos y Disensos. Ridu, 13(1), 101122, e-ISSN: 2223-2516. Recuperado de:

http://dx.doi.org/10.19083/ridu.2019.644 
Thorndike, E. (1920). Intelligence and its uses. Harper's Magazine, 140, 227 235. New York, United States: Harper's Magazine Foundation.

Yin, R. (2013). Case Study Research: Design and Methods (Applied Social

Research Methods). Fifth Edition, ISBN: 978-1452242569. United States: SAGE Publications, Inc. 
OAI-PMH: http://www.indteca.com/ojs/index.php/Revista_Scientific/oai

Artículo Original / Original Article

\section{María Auxiladora Campos Medina}

e-mail: maríauxicamposmedina@hotmail.com

Nacida en Barquisimeto, Venezuela, el 25 de febrero del año 1966. Realicé mis estudios de pregrado en la Universidad Nacional Abierta (UNA), donde obtuve el título de Licenciada en Dificultades del aprendizaje; soy Magíster en Orientación; obtuve el doctorado en Ciencias con especialidad en Psicología por la Atlantic International University (AIU); Doctora en Gerencia por la Universidad Yacambú (UNY); Académica en la Universidad Nacional Abierta UNA), la Universidad Yacambú (UNY) y la Universidad Centroccidental Lisandro Alvarado (UCLA); Coordinadora de Cultura en la Universidad Nacional Abierta (UNA); Centro Local Lara; Otros títulos: Training Practitioner en Programación Neurolingüística por la Universidad Fermín Toro (UFT); Diplomado de Experto en E-learning por la Fundación para la actualización tecnológica de Latinoamérica (FATLA); Premio Camino a la Excelencia de la Universidad Nacional Abierta (UNA); Entre las investigaciones que ha realizado se nombran: Inteligencia Emocional y rendimiento académico en la Universidad Centroccidental Lisandro Alvarado (UCLA); Relación entre el estrés, la inteligencia emocional y el rendimiento académico en la UCLA; Ponente en diversos Congresos.

El contenido de este manuscrito se difunde bajo una Licencia de Creative Commons ReconocimientoNoComercial-Compartirlgual 4.0 Internacional 